\title{
ON INTEGRALS RELATED TO AND EXTENSIONS OF THE LEBESGUE INTEGRALS.
}

BY PROFESSOR T. H. HILDEBRANDT.

(Read at the Chicago Symposium of the American Mathematical Society, April 6, 1917.)

IN the theory of definite integration, the central position is occupied by the definitions of integration due to Riemann and to Lebesgue. There have however been given a number of other definitions, some of which are related to and in part equivalent to the Lebesgue integral, and some of which are extensions of it; viz., in the direction of giving an integral for a function for which the Lebesgue definition is not applicable either on account of the fact that the set of points at which it is defined is not measurable, or on account of the fact that the function is not absolutely integrable; and in the direction of integration with respect to functions of bounded variation. We purpose in this paper to discuss briefly some of the definitions of integration which have been proposed and consider their relations to the Lebesgue integral. We shall divide the work into four sections.

In the first section we discuss the types of definition of integration which are extensions of the Darboux upper and lower integral method of defining a Riemann integral. Essentially the process involves three steps, $(a)$ the selection of a simple class of functions and the definition of integration for functions of this class, $(b)$ the extension of this class of functions by the addition of functions which are the limits of sequences converging either uniformly or monotonically, the integral of the limit being defined to be the limit of the integrals, $(c)$ the further extension of this class by the addition of all functions $f(x)$ for which there exist functions $\varphi$ and $\psi$ belonging to the classes defined in $(a)$ or $(b)$, with equal definite integrals, and such that $\varphi \leqq f \leqq \psi$. We find ideas of this kind in the Lebesgue integration, the last of these steps suggesting, in particular, the distinguishing feature between Borel and Lebesgue measurable sets. Step (b), when the convergence is uniform, is present in the Lebesgue definition, while, when we take rather the monotonic sequence, we have 
the ideas underlying the Young definition which we consider here. This definition is, as we shall show, equivalent to that of Lebesgue in the sense that every function integrable according to either definition is integrable according to the other and the values of the integrals are the same. Very much of the same order is the definition due to Pierpont, which differs from the definition of Young only in that it gives an integral for functions defined on non-measurable sets-assuming that such sets exist. We point out that the Pierpont integral is an extension only in a limited way, in that the functions which are integrable according to this definition are non-measurable in the sense that they are defined on non-measurable sets; in particular we show that if $f$ is Pierpont integrable on a non-measurable set $E$ then there exists a measurable set $M$ containing $E$ and a function $\varphi$ defined on $M$ which is equal to $f$ on $E$, such that the Lebesgue integral of $\varphi$ on $M$ is the same as the Pierpont integral of $f$ on $E$. Perhaps by stretching the meaning of the word equivalence a little, we might say that the Pierpont and Lebesgue integrals are equivalent.

The second section is devoted to a consideration of the definitions of the integrals of functions which are not integrable according to Lebesgue's definition, because the latter are always absolutely integrable. We consider two types of definitions. The first type depends upon the ability to find a set of intervals whose total length may be made to approach zero, and such that it is possible to define an integral for the function when defined only on the complementary set of points. If these latter integrals approach a limit as the total length of the complementary intervals approaches zero, the limit is the integral over the interval. The integral is thus defined as a result of a single limiting process, and all the singularities are treated simultaneously. The definitions of this type are the Jordan-Harnack-Moore definitions, in which the enclosing intervals are finite in number, and the Borel definitions, in which the intervals may be denumerably infinite in number, i. e., the first are based on content and the second on measure. The other type of definition depends upon the arrangement of the singularities of the function to be integrated in some order, the arrangement and the singularities being dependent upon the integration process used, the ultimate value of the integral being obtained by at most a denumerable infinity of steps and limiting processes. In this cate- 
gory belong the Dirichlet extension of the Cauchy integral for unbounded functions, and the extension of this to the case when the points in the vicinity of which $f(x)$ is not bounded form a reducible set. It is this idea also which Denjoy has applied successfully in order to obtain a definition of integral of unbounded functions which includes as special cases practically all those which have been previously suggested, an integral which proves of importance in the treatment of the relation between a continuous function and its derivative.

The third section is devoted to the Stieltjes integral, which has recently come into the foreground on account of the rôle which it plays in the theory of linear functional operations on continuous functions. We point out that a Stieltjes integral is expressible in terms of a Lebesgue integral of another function, and conversely, but that in spite of this, the Stieltjes integral seems to be applicable where the Lebesgue is not. We give an extension of the Stieltjes integral modelled on the Lebesgue extension of the Riemann integral, as well as the Fréchet generalization of the Lebesgue and Stieltjes integral, so as to apply to a class of general elements. This latter integral depends for its definition on the existence of an absolutely additive function whose range is a set of subclasses of the fundamental class.

The last section gives the definition of the Hellinger integral and also the generalizations of this due to Radon and E. H. Moore.

In the course of the paper we have occasion to point out certain equivalences. They are of two types. The first might be called a complete equivalence, i. e., one in which two definitions of integration yield the same class of integrable functions, and give the same value when applied to a given function. Of this character are the equivalence between the Young and Lebesgue, and one of the Borel definitions and that of Lebesgue;--and we might call attention in passing to what seems. to be an indication of the felicity of the Lebesgue definition, that it can be approached from so many distinct and interesting points of view. The other type of equivalence is that which arises in connection with the Stieltjes and Hellinger integrals, each of which is by a transformation reducible to a Lebesgue integral, and conversely; but in either case the function to be integrated is no longer the same. We are inclined to consider this type of equivalence in the light of a 
pseudo-equivalence, in so far as it is rather a reduction from one form to the other for purposes of evaluation.

The extension of the Lebesgue integral idea in the future will undoubtedly be in the direction of the formulation of a satisfactory integral operation for the case in which the field of integration is a space of a denumerable infinity of dimensions, and the function space. Thus far, there have been given two suggestions for such operations. One is due to Fréchet, who forms, for a general class, the natural extension of the Lebesgue and Stieltjes definition of integration. It depends upon the existence, in a general class of elements, of an absolutely additive function $v$ whose range is a class of subclasses of the fundamental class, i. e., a function such that

$$
v\left(\Sigma_{n} E_{n}\right)=\Sigma_{n} v\left(E_{n}\right)
$$

the $E_{n}$ being mutually distinct and finite or denumerably infinite in number. The examples of this which have been given for the general space are trivial in that they reduce either to an infinite sum or an integral extended over a field in a finite number of dimensions. There is still lacking a really effective and desirable absolutely additive function for the higher type of spaces. The other suggestion for generalization is due to $\mathrm{E}$. H. Moore. It is essentially an operator of the bilinear or quadratic type, the Fréchet generalization being of the linear type. It depends upon the existence of a function $\epsilon(p, q)$ of two variables $p$, $q$, each of which ranges independently over the fundamental class of general elements, and satisfies the condition that for any finite set of elements $p_{1}, \cdots, p_{n}$, the quantities $\epsilon\left(p_{i}, p_{j}\right)$, $i, j=1, \cdots, n$, form the coefficients of a positively definite Hermitian form in $n$ variables. The operator is determined as a double least upper bound, and is theoretically given as soon as the proper type of $\epsilon$ is specified. Moore has given instances of this $\epsilon$ in various general spaces which are not trivial. While these two generalizations point the way in which one may go towards the generalization of the Lebesgue integral, they are not entirely satisfactory, the Fréchet generalization, as indicated above, because his instances are trivial, the Moore because it reduces to a Lebesgue integral only after a transformation. There is still room for considerable improvement and investigation in this field.

We restrict ourselves throughout this paper to functions 
which are single-valued and take finite values at points belonging in most cases to a finite interval $(a, b)$, or a set of points on such an interval. The functions are not, however, necessarily bounded on the interval $(a, b)$. Many of the results are immediately extensible to fields of integration in $n$-dimensional space. Further, some of the theorems are still valid if $f(x)$ be definitely infinite or many-valued at a set of points of measure zero.

For the sake of convenience we have collected here the most important references which we have consulted in the compilation of this paper, and shall cite them only by number.

1. BuIss, G. A. "Integrals of Lebesgue," BuLletin of the American Mathematical Society, vol. 24 (1917), pp. 1-47.

2. Borel, E. (a) "Le calcul des intégrales définies," Journal de Mathématiques, ser. 6, vol. 8 (1912), pp. 159-210; (b) La théorie de la mesure et la théorie de l'intégration, Leçons sur la Théorie des Fonctions, 2d edition (1914), pp. 217-256.

3. Denjoy, A. (a) "Une extension de l'intégrale de M. Lebesgue," Comptes Rendus, vol. 154 (1912), pp. 859-862; (b) "Calcul de la primitive de la fonction dérivée la plus générale," ibid., pp. 1075-8.

4. Denjoy, A. "Sur la dérivation et son calcul inverse," (a) Journal de Mathématiques, ser. 7, vol. 1 (1915), pp. 105-240; (b) Bulletin de la Société Mathématique de France, vol. 43 (1915), pp. 161-249; (c) Annales de l'Ecole Normale Supérieure, vol. 33 (1916), pp. 127-223.

5. Frfснет, M. "Sur les fonctionnelles linéaires et l'intégrale de Stieltjes," Comptes Rendus du Congrès des Sociétés savantes en 1913, pp. 45-54.

6. FrÉcheт, M. "Sur l'intégrale d'une fonctionnelle étendue à un ensemble abstrait," Bulletin de la Société Mathématique de France, vol. 43 (1915), pp. 249-267.

7. HАнN, H. "Ueber die Integrale des Herrn Hellinger," Monatshefte für Mathematik und Physik, vol. 23 (1912), pp. 161-224.

8. HAHN, H. "Ueber eine Verallgemeinerung der Riemannschen Integraldefinition," Monatshefte für Mathematik und Physik, vol. 26 (1915), pp. 3-18.

9. HarnacK, A. "Die allgemeinen Sätze über den Zusammenhang der Funktionen einer reellen Variabeln mit ihren Ableitungen," Mathematische Annalen, vol. 24 (1884), pp. 217-252.

10. Hausdorff, F. Grundzüge der Mengenlehre (1914), pp. $408 \mathrm{ff}$.

11. Hellinger, E. "Neue Begründung der Theorie quadratischer Formen von unendlichvielen Variabeln," Journal für Mathematik, vol. 136 (1909), pp. 210-271.

12. Jordan, C. Cours d'Analyse, $2 \mathrm{~d}$ edition, vol. II, pp. 50, 51.

13. Lebesgue, H. Leçons sur l'Intégration (1904).

14. Lebesgue, H. "Sur l'intégrale de Stieltjes et sur les opérations linéaires," Comptes Rendus, vol. 150 (1910), pp. 86-88. 
15. Moore, E. H. "Concerning Harnack's theory of improper definite integrals," Transactions of the American Mathematical Society, vol. 2 (1901), pp. 296-330. See also pp. 459-475.

16. Nalim, P. Esposizione e confronto critico delle diverse definizioni proposte per l'integrale definito di una funzione limitata o no. Palermo thesis (1914).

17. Perron, O. Die Lehre von den Kettenbrüchen (1913), pp. 362-374.

18. Pierpont, J. Theory of Functions of Real Variables, vol. II (1912) pp. $343 \mathrm{ff}$.

19. Radon, J. "Absolut additive Mengenfunktionen," Wiener Sitzungsberichte, vol. $122_{2 a}$ (1913), pp. 1295-1438.

20. RIEsz, F. "Systeme integrierbarer Funktionen," Mathematische Annalen, vol. 69 (1910), pp. 449-497.

21. RiEsz, F. "Sur certains systèmes singuliers d'équations intégrales," Annales de l'École Normale Supérieure, ser. 3, vol. 28 (1911), pp. 33-62.

22. RIEsz, F. "Les opérations fonctionnelles linéaires," Annales de l'École Normale Supérieure, ser. 3, vol. 31 (1914), pp. 9-14.

23. Stieltues, T. J. "Sur les fractions continues," Annales de la Faculté des Sciences de Toulouse, vol. 8 (1894), pp. J71 ff.

24. de la Valcter Poussin, C. J. Intégrales de Lebesgue, Fonctions d'Ensemble, Classes de Baire, Paris 1916. Cf. also Transactions of the American Mathematical Society, vol. 17 (1916), pp. 435-501.

25. VAN VLECK, E. B. "Haskins' momental theorem and its connection with Stieltjes's problem of moments," Transactions of the American Mathematical Society, vol. 18 (1917), pp. 326-330.

26. Young, W. H. "On the general theory of integration," Philosophical Transactions, vol. $204 A$ (1905), pp. 221-252.

27. Young, W. H. "On the new theory of integration," Proceedings of the Royal Philosophical Society, vol. 88A (1913), pp. 170-178.

28. YounG, W. H. "On a new method in the theory of integration," Proceedings of the London Mathematical Society, ser. 2, vol. 9 (1910), pp. 15-50.

29. Young, W, H. "On integration with respect to a function of bounded variation," Proceedings of the London Mathematical Society, ser. 2, vol. 13 (1914), pp. 109-150.

\section{The Young and Pierpont Definitions of INTEGRATION.}

1. The Darboux Integrals.-In so far as the Young and Pierpont definitions of integration which we consider in this chapter are extensions of the Darboux treatment of the Riemann integral by means of upper and lower integrals, we recall briefly the definition of these integrals for functions $f(x)$ bounded on an interval $(a, b)$ and a few of their properties.

Definition.-Suppose a partition of the interval $(a, b)$ has been effected by means of the points $a=x_{0}<x_{1}<\cdots<x_{n}=b$. 
Let $M_{i}$ be the least upper bound and $m_{i}$ the greatest lower bound of the values of $f(x)$ on $\left(x_{i-1}, x_{i}\right)$ and form the sums

$$
S=\sum_{i=1}^{n} M_{i}\left(x_{i}-x_{i-1}\right) \quad \text { and } \quad s=\sum_{i=1}^{n} m_{i}\left(x_{i}-x_{i-1}\right) .
$$

Then the greatest lower bound of $S$ and the least upper bound of $s$ exist, if we consider all possible partitions of the interval $(a, b)$ into a finite number of subintervals. These bounds are the upper and lower Darboux integrals, respectively, of $f(x)$ on $(a, b)$ and are denoted by

$$
\overline{\int_{a}^{b}} f(x) d x \quad \text { and } \quad \int_{a}^{b} f(x) d x .
$$

We note the following properties:

(1) If $m \leqq f(x) \leqq M$ for $x$ on $(a, b)$, then

$$
m(b-a) \leqq s \leqq \int_{a}^{b} f(x) d x \leqq \int_{a}^{b} f(x) d x \leqq S \leqq M(b-a) .
$$

(2) The value of $S$ is not increased, and that of $s$ is not diminished by a repartition of a partition of $(a, b)$.

(3) There exists a sequence of partitions of $(a, b)$ each a repartition of the preceding and with norms approaching zero such that, if $s_{n}$ and $S_{n}$ are the corresponding sums $s$ and $S$, we have

$$
\lim _{n} s_{n}=\int_{a}^{b} f(x) d x \quad \text { and } \quad \lim _{n} S_{n}=\bar{\int}_{a}^{b} f(x) d x .
$$

Evidently $s_{1} \leqq s_{2} \leqq \cdots$ and $S_{1} \geqq S_{2} \geqq \cdots$. Moreover if $\varphi_{n}(x)=M_{n, i}$ and $\psi_{n}(x)=m_{n, i}$ in the $i$ th interval $\left(x_{i-1}\right.$ $\left.\leqq x<x_{i}\right)$ of the $n$th partition, then $\lim _{n} \varphi_{n}=\varphi$ and $\lim _{n} \psi_{n}=\psi$ exist and we have

$$
\psi \leqq f \leqq \varphi ; \quad \text { and } \quad \bar{\int} \varphi=\bar{\int} f \quad \text { and } \quad \underline{\int} \psi=\underline{\int} f .
$$

(4) A necessary and sufficient condition that $f(x)$ be Riemann integrable is that $\underline{\int}=\bar{\int}=\int$.

From this, other necessary and sufficient conditions for Riemann integrability can be deduced. Perhaps the simplest is the following, due to Lebesgue ((13), page 29): 
(5) A necessary and sufficient condition that $f(x)$ be Riemann integrable is that the points of discontinuity of $f(x)$ form a set of measure zero.

2. The Young Definition of Integration.-(Cf. Young (26), (27), (28); Nalli (16), pages 97-103.) The Riemann-Darboux definitions of integration depend upon a division of the interval of integration into subintervals; the Lebesgue definition on a division of the interval of variation of the function, which for the interval of integration results in a partition into measurable sets. The question naturally arises whether it is possible to find a definition of Lebesgue integral by replacing in the Darboux definition intervals by measurable sets.* Young ((26), page 243) therefore suggests the following

Definition. Divide the interval $(a, b)$ into a finite or $a$ denumerably infinite number of measurable sets $E_{i}$ of measure $d_{i}$. Let $M_{i}$ be the least upper bound and $m_{i}$ the greatest lower bound of $f(x)$ on $E_{i}$ and form the sums

$$
S=\Sigma_{i} M_{i} d_{i} \quad \text { and } \quad s=\Sigma_{i} m_{i} d_{i} .
$$

Then the greatest lower bound of $S$ and the least upper bound of $s$ for all possible divisions of $(a, b)$ into measurable sets are defined to be the Young (or (Y)) upper and lower integrals of $f(x)$ on $(a, b)$. $f(x)$ is said to be Young (or $(Y)$ ) integrable if the upper and lower integrals are finite and equal, $i . e$. ,

$$
(Y) \mathcal{J}=(Y) \underline{\mathcal{J}}=(Y) \overline{\boldsymbol{J}} \text {. }
$$

Obviously this definition is also applicable in case the interval $(a, b)$ is replaced by any measurable set of points $E$.

This definition was originally suggested only for functions $f(x)$ bounded on $(a, b)$. In that case the upper and lower integrals always exist, and we obtain the same value for each of them, whether the partition be into a finite or a denumerably infinite number of measurable sets. The same definition will apply also if $f(x) \dagger$ is not bounded, provided we assume that $f(x)$ is such that there exist partitions of $(a, b)$ into a denumerable infinity of measurable sets on each of which $f(x)$

* De la Vallee Poussin (24), pp. 54-56, has pointed out that the Riemann integral is characterized by being a function of intervals, the Lebesgue integral being a function of measurable sets.

$\uparrow$ While not expressed explicitly in Young's work, it seems that he considered this extension. Cf., for instance, (28), p. 35. 
has a finite upper and lower bound. In determining the upper and lower integrals in this case, we restrict ourselves to partitions of this latter kind.

We are then able to show that, if the upper integral has not the value $-\infty$, the terms of the sum $\Sigma_{i} M_{i} d_{i}$, in which $M_{i}$ is negative, form a convergent series, i. e., for every such partition, $\Sigma_{i} M_{i} d_{i}$ either diverges to $+\infty$ or converges absolutely. For, if the negative terms of this sum are divergent, then by suitable repartitions of $(a, b)$ and rearrangement of the terms, we can make the sum approach $-\infty$. An analogous result holds for the lower integral, i. e., if it is not $+\infty$, then it either diverges to $-\infty$ or converges. It follows from this that a necessary and sufficient condition that the upper and lower integrals of $f(x)$ be finite is that there exist a partition of $(a, b)$ such that if we form the sum $\Sigma_{i} M_{i} d_{i}$ for $|f(x)|$ this sum is convergent.*

In the following list of properties we exclude the case in which $(Y) \bar{\int}=-\infty$ or $(Y) \underline{\int}=+\infty$. Then the proofs are very much as in the case of the Darboux integrals, in so far as the series which enter will be absolutely divergent or convergent. We have

$$
\text { (Y) } \bar{\int} \geqq(Y) \underline{\int} .
$$

If $f(x)$ is bounded, i. e., $m \leqq f \leqq M$, for every $x$ on $(a, b)$. then

$$
m(b-a) \leqq(D) \underline{\int} \leqq(Y) \underline{\int} \leqq(Y) \bar{\int} \leqq(D) \bar{\int} \leqq M(b-a) .
$$

(2) The values of $S$ are not increased, nor those of $s$ diminished by a repartition of a partition of $(a, b)$ into measurable sets.

(3) There exists a sequence of partitions of $(a, b)$ into measurable sets, each a repartition of the preceding, such that if $S_{n}$ and $s_{n}$ are the corresponding values of $S$ and $s$, then

and

$$
\lim _{n} S_{n}=(Y) \bar{\int} \text { and } \lim _{n} s_{n}=(Y) \underline{\int}
$$

$$
S_{1} \geqq S_{2} \geqq \cdots \quad \text { and } \quad s_{1} \leqq s_{2} \leqq \cdots .
$$

If we let $\varphi_{n}(x)=M_{n, i}$ and $\psi_{n}(x)=m_{n, i}$ on the $i$ th set of

\footnotetext{
* Cf. Fréchet (6), p. 257.
} 
the $n$th partition, then the functions $\varphi_{n}(x)$ will form a monotonic non-increasing sequence and the $\psi_{n}(x)$ a monotonic non-decreasing sequence of measurable functions such that

$$
\lim _{n} \psi_{n}(x)=\psi(x) \leqq f(x) \leqq \varphi(x)=\lim _{n} \varphi_{n}(x) .
$$

Evidently the Lebesgue integrals of $\varphi_{n}(x)$ and $\psi_{n}(x)$ exist and are equal to the values of $S_{n}$ and $s_{n}$ respectively. Hence by the properties of Lebesgue (or $(L)$ ) integrals relative to monotonic sequences (cf. (13), page 98), we have

$$
\lim _{n} S_{n}=\lim _{n}(L) \int \varphi_{n}=(L) \int \varphi=(Y) \bar{\int} f
$$

and

$$
\lim _{n} s_{n}=\lim _{n}(L) \int \psi_{n}=(L) \int \psi=(Y) \underline{\int} f .
$$

3. The Relation between the Young and Lebesgue Integrals.(Cf. Young (26), pages $243 \mathrm{ff.;}$ Nalli (16), pages 97-106.) A direct consequence of (1) of $\$ 2$ is that if $f(x)$ is Riemann integrable, it is also Young integrable. More generally we have:

The Young and Lebesgue definitions of integration are equivalent, and the values obtained by the two definitions the same.

(a) Suppose $f(x)(L)$ integrable on $(a, b)$. Let $l_{i}(i=-\infty$ to $+\infty)$ be points of division of the interval $(-\infty,+\infty)$. Then the sum

$$
\sigma=\Sigma_{i} l_{i} \times \operatorname{meas}\left(E\left(l_{i} \leqq f<l_{i+1}\right)\right)
$$

will be an $s$ and the sum

$$
\Sigma=\Sigma_{i} l_{i} \times \operatorname{meas}\left(E\left(l_{i-1}<f \leqq l_{i}\right)\right)
$$

will be an $S$. Hence

$$
\sigma \leqq(Y) \underline{\int} \leqq(Y) \bar{\int} \leqq \Sigma .
$$

Since $\sigma$ and $\Sigma$ approach the $(L) \int$ as the maximum value of the difference $l_{i+1}-l_{i}$ approaches zero, it is apparent that

$$
\text { (L) } \int=(Y) \underline{\mathcal{J}}=(Y) \overline{\boldsymbol{J}}=(Y) \int \text {. }
$$

(b) On the other hand suppose that $f(x)$ is $(Y)$ integrable on the interval $(a, b)$. Then by property (3) of $\S 2$ 


$$
\text { (L) } \int \psi=(Y) \int f=(Y) \int f=(Y) \bar{\int} f=(L) \int \varphi,
$$

i. e., $(L) \int(\varphi-\psi)=0$. Hence $\varphi$ differs from $\psi$ at most at a set of points of measure zero, and consequently, since $\psi \leqq f \leqq \varphi$ at every point, $f$ differs from the measurable and $(L)$ integrable functions $\varphi$ and $\psi$ at most at a set of measure zero, and hence is measurable and also $(L)$ integrable.

Closely allied with the idea used in this last proof is the following definition of Lebesgue integration due to Riesz ((20), page 453).

The integral is defined first of all for functions which are constant on measurable sets of points. If $f(x)$ is equal to $a_{i}$ on the set $E_{i}$ of measure $d_{i}$, then

$$
\int_{a}^{b} f(x) d x=\Sigma_{i} a_{i} d_{i}
$$

the series on the right being assumed to be absolutely convergent if infinite. If we extend this class of functions by the addition of all limits of uniformly convergent sequences of such functions, we get the class of Lebesgue integrable functions. The integral of the limiting function is of course, by definition, the limit of the integrals. This definition lends itself admirably to the proof of theorems of the nature of the Schwarz inequality for summable functions.

In reality the above proof suggests the extension of the class of functions constant on measurable subsets of $(a, b)$ by the addition of the limits of monotonic sequences of these functions, together with the functions which lie between a limit $\varphi$ of a non-decreasing sequence, and a limit $\psi$ of a nonincreasing sequence for which the integrals are equal in value. Young (cf. (27) and (28)) has developed the theory of Lebesgue integration on the basis of monotonic sequences as just sketched, excepting that the initial functions for which integration is defined are upper and lower semi-continuous.

4. Excursus on Upper and Lower Measure.-(Cf. Hausdorff (10), pages 408-411.) Before taking up the discussion of the Pierpont integral it may be advantageous to derive a few elementary properties relating to upper and lower measure. We recall that the upper measure of a set is the greatest lower bound of the total lengths of the sets of open intervals which are such that each interval contains at least one point 
of the set; that the lower measure is the measure of an interval containing the set, diminished by the upper measure of the complementary set with respect to the interval.

If for two sets $E_{1}$ and $E_{2}$ we denote by $E_{1}+E_{2}$ their least common superset, and by $E_{1} E_{2}$ their greatest common subset, i. e., their logical sum and product, respectively, we have the following fundamental formula on intervals $I_{1}, I_{2}$ :

(1) meas $\left(I_{1}+I_{2}\right)+$ meas $I_{1} I_{2}=$ meas $I_{1}+$ meas $I_{2}$,

which is immediately extensible to a finite or a denumerably infinite set of intervals. An immediate consequence of this formula is

(2) $\overline{\text { meas }}\left(E_{1}+E_{2}\right)+\overline{\text { meas }} E_{1} E_{2} \leqq \overline{\text { meas }} E_{1}+\overline{\text { meas }} E_{2}$

and by taking complements with respect to an interval including both $E_{1}$ and $E_{2}$

(3) meas $\left(E_{1}{ }^{\prime}+E_{2}{ }^{\prime}\right)+$ meas $E_{1}{ }^{\prime} E_{2}{ }^{\prime} \geqq$ meas $E_{1}{ }^{\prime}+$ meas $E_{2}{ }^{\prime}$, where $E_{1}{ }^{\prime}=C E_{1}$ and $E_{2}{ }^{\prime}=C E_{2}$; i. e., this formula holds for any $E_{1}$ and $E_{2}$. From these two inequalities we conclude at once that if $E_{1}$ and $E_{2}$ are measurable, then the sets $E_{1}+E_{2}$ and $E_{1} E_{2}$ are also measurable and

(4) meas $\left(E_{1}+E_{2}\right)+$ meas $E_{1} E_{2}=$ meas $E_{1}+$ meas $E_{2}$.

From (2) we conclude for every $E_{1}$ and $E_{2}$

(5) $\overline{\text { meas }}\left(E_{1}+E_{2}\right) \leqq \overline{\text { meas }} E_{1}+\overline{\text { meas }} E_{2}$;

and from (3), if $E_{1}$ and $E_{2}$ have no points in common,

(6) meas $\left(E_{1}+E_{2}\right) \geqq$ meas $E_{1}+$ meas $E_{2}$.

Further, since for every $E_{1}$ and $E_{2}$

$$
C E_{1} \leqq C\left(E_{1}+E_{2}\right)+E_{2},
$$

we obtain by applying (5)

or

$$
\overline{\text { meas }} C E_{1} \leqq \overline{\text { meas }} E_{2}+\overline{\text { meas }} C\left(E_{1}+E_{2}\right) \text {, }
$$

(7) meas $E_{1}+\overline{\text { meas }} E_{2} \geqq$ meas $\left(E_{1}+E_{2}\right)$.

Similarly if $E_{1}$ and $E_{2}$ are distinct, we can apply (6) to

$$
C E_{1}=C\left(E_{1}+E_{2}\right)+E_{2}
$$


and obtain

(8) $\overline{\text { meas }} E_{1}+$ meas $E_{2} \leqq \overline{\text { meas }}\left(E_{1}+E_{2}\right)$.

If, then, $E_{1}$ and $E_{2}$ are distinct, we can write down the following series of inequalities:

(9) meas $E_{1}+$ meas $E_{2} \leqq$ meas $\left(E_{1}+E_{2}\right) \leqq$ meas $E_{1}$

$$
\begin{aligned}
& +\overline{\text { meas }} E_{2} \\
& \leqq \overline{\text { meas }}\left(E_{1}+E_{2}\right) \leqq \overline{\text { meas }} E_{1} \\
& +\overline{\text { meas }} E_{2} \text {. }
\end{aligned}
$$

5. Relative Measurability; Separated Sets.-(Cf. Hausdorff (10), pages 415-6; Pierpont (18), pages 366-370.) We define:

$E_{0}$ will be said to be measurable relative to $E$, if there exists a measurable set $M$ such that $E_{0}=M E$, i. e., $E_{0}$ is, so to speak, a section of the set $E$ by a measurable set.

Evidently $E$ is measurable relative to itself. Also, if $E_{0}$ is measurable relative to $E$, then $E-E_{0}$ will also be measurable relative to $E$. Finally by (4) of $\S 4$, we have that if $E$ is measurable, then relative measurability reduces to ordinary measurability.

We note the following propositions:

(1) If $E$ is divided into a finite or a denumerable infinity of sets $E_{i}$ measurable relative to $E$, then

$$
\overline{\text { meas }} E=\Sigma_{i} \overline{\text { meas }} E_{i} \quad \text { and } \quad \text { meas } E=\Sigma_{i} \text { meas } E_{i} \text {. }
$$

For the case of two sets $E_{1}$ and $E_{2}$ with $E_{1}+E_{2}=E$ and $E_{1}=M E$, where $M$ is a measurable set, we have by (2) of $\S 4$ $\overline{\text { meas }} M+\overline{\text { meas }} E \geqq \overline{\text { meas }}(M+E)+\overline{\text { meas }} M E$

and so by (8) of $\S 4$

$$
=\overline{\text { meas }}\left(M+E_{2}\right)+\overline{\text { meas }} E_{1} \text {, }
$$

$$
\geqq \text { meas } M+\overline{\text { meas }} E_{2}+\overline{\text { meas }} E_{1} \text {. }
$$

Since $M$ is measurable, we obtain by using (5) of $\S 4$

$$
\overline{\text { meas }} E=\overline{\text { meas }} E_{1}+\overline{\text { meas }} E_{2} \text {. }
$$

The property for lower measure can be obtained in an analogous way by using (3), (7), and (6) of $\S 4$. The corresponding 
properties for any finite number, or denumerable infinitude of sets, follow at once from a recurrent use of the result for the case of two sets, and the properties

$$
\overline{\text { meas }} \Sigma E_{i} \leqq \Sigma_{i} \overline{\text { meas }} E_{i} \text {; }
$$

and if $\lim _{n} E_{n}=0$, then $\lim _{n}$ meas $E_{n}=0$.

The following converse of this proposition holds:

(2) If $E$ is divided into a finite or denumerable set of sets $E_{i}$ for which we have $\overline{\text { meas }} E=\Sigma_{i} \overline{\text { meas }} E_{i}$, then the $E_{i}$ are measurable relative to $E$ and meas $E=\Sigma_{i}$ meas $E_{i}$.

We indicate the proof for two sets only, the case for a denumerably infinite number of sets being easily deducible from this. Suppose $E=E_{1}+E_{2}, E_{1}$ and $E_{2}$ being distinct, and suppose $M_{1}$ and $M_{2}$ are measurable sets such that $E_{1}$ is contained in $M_{1}$ and $E_{2}$ in $M_{2}$, and meas $M_{1}=\overline{\text { meas }} E_{1}$ and meas $M_{2}=\overline{\text { meas }} E_{2}$. Then by (4) of $\S 4$

$$
\text { क्meas } E=\text { meas } \begin{aligned}
M_{1} & + \text { meas } M_{2}=\text { meas }\left(M_{1}+M_{2}\right) \\
& + \text { meas } M_{1} M_{2} \geqq \overline{\text { meas }} E+\text { meas } M_{1} M_{2},
\end{aligned}
$$

i. e., meas $M_{1} M_{2}=0$. Hence $\overline{\text { meas }} M_{2} E_{1}=0$, i. e., $M_{2} E_{1}$ is measurable. Consequently, since

$$
\left(M_{2}-M_{2} E_{1}\right) E=M_{2} E-M_{2} E_{1}=E_{2},
$$

$E_{2}$ will be the set common to $E$ and the measurable set $M_{2}-M_{2} E_{1}$ and so measurable relative to $E$.

As a consequence of these two propositions we have

(3) If $E_{1} \leqq E_{2} \leqq \cdots$ are a sequence of sets, each containing the preceding, and each measurable relative to the succeeding set in the sequence, then every set as well as the difference of any two sets, will be measurable relative to $E=\lim _{n} E_{n}$.

(4) If $M$ is a measurable set containing $E$ such that meas $M=\overline{\text { meas }} E$, then for any division of $E$ into sets measurable relative to $E$, there exists a division of $M$ into measurable sets $M_{i}$ such that

$$
\text { meas } M_{i}=\overline{\text { meas }} E_{i} \quad \text { and } \quad E_{i}=M_{i} E .
$$

Pierpont ((18), page 366) uses in his work the notion of separated division, defined as follows:

$E$ is divided into the separated sets $E_{1}$ and $E_{2}$ if it is 
possible to enclose $E_{1}$ and $E_{2}$ in measurable sets $M_{1}$ and $M_{2}$ in such a way that meas $M_{1} M_{2}=0 . *$

Pierpont ((18), page 379) shows that if $E_{1}$ and $E_{2}$ are separated then

$$
\overline{\text { meas }} E=\overline{\text { meas }} E_{1}+\overline{\text { meas }} E_{2} \text {. }
$$

By using proposition (2) and its proof, we then have:

A necessary and sufficient condition that $E_{1}$ and $E_{2}$ constitute a separated division of $E$ is that $E_{1}$ and $E_{2}$ be measurable relative to $E$, or that

$$
\overline{\text { meas }} E_{1}+\overline{\text { meas }} E_{2}=\overline{\text { meas }}\left(E_{1}+E_{2}\right)=\overline{\text { meas }} E \text {. }
$$

Consequently, we have also

$$
\text { meas } E_{1}+\underline{\text { meas }} E_{2}=\underline{\text { meas }} E \text {. }
$$

The same result holds if the division is into a finite or denumerably infinite number of separated sets.

Evidently, then, if $E$ is measurable, any separated division of $E$ is always into measurable sets. $\dagger$

We shall use the term relatively measurable in preference to separated, on account of the close relationship to measurable sets.

6. The Pierpont Definition of Integration.-(Cf. Pierpont (18), pages $371 \mathrm{ff}$.) We are now in a position to give the Pierpont extension of the Young definition of integration.

Suppose $E$ is any set of points on a finite interval, and $f(x)$ defined on $E$. Divide $E$ into a finite or denumerable set of sets $E_{i}$ measurable relative to $E$, and let $M_{i}$ be the least upper bound, and $m_{i}$ the greatest lower bound of $f(x)$ on $E_{i}$. Form the sums $S=\Sigma_{i} M_{i}$ meas $E_{i}$ and $s=\Sigma_{i} m_{i}$ meas $E_{i}$. The greatest lower bound of $S$ and the least upper bound of $s$ for all possible divisions of $E$ into sets measurable relative to $E$ are the upper and lower integrals, respectively, of $f(x)$ on $E . f(x)$ is said to be $(P)$ integrable on $E$ if these two integrals are finite and equal, $i$. e.,

$$
(P) \bar{\int}_{E}=(P) \int_{E}=(P) \int_{E} \text {. }
$$

* We shall assume that $E_{1}$ and $E_{2}$ are distinct. Pierpont allows $E_{1}$ and $E_{2}$ to overlap, but since meas $M_{1} M_{2}=0$ we have also meas $E_{1} E_{2}=0$, i. e., they overlap only to the extent of a set of measure zero. The results obtained are the same with either definition, the treatment being slightly simpler as here given.

$\dagger$ It seems that recognition of this fact would have obviated the recent Fréchet-Pierpont controversy in these columns, vol. 22, 295-302; vol. 23, 172-5. Cf. also W. A. Wilson, vol. 22, pp. 384-386. 
Evidently when the set $E$ is measurable this definition is identical with that of Young. Although stated only for bounded functions by Pierpont, it is immediately applicable to functions not bounded on $E$. As a matter of fact the remarks made in connection with the Young integral can be immediately transferred to the Pierpont integral if we replace measurable by relatively measurable, and measure by upper measure. In particular, if we say that $f(x)$ is measurable relative to $E$ (Pierpont, separable, cf. (18), page 403), if the set $E_{l}$ of points of $E$ for which $f(x)>l$ is measurable relative to $E$, we can build up an integral along the lines of Lebesgue. If we call a function integrable in this way summable relative to $E$, we have the theorem:

(1) Pierpont integrability on $E$ is equivalent to summability relative to $E$, the values obtained for the two integrals being the same.*

We note further the property

(2) If $E$ is divided into a set of sets $E_{i}$ measurable relative to $E$, and if $f$ is integrable on each $E_{i}$, then $f$ is also integrable on $E$ and $\int_{E}=\Sigma_{i} \int_{E_{i}}$

On the other hand we have the following:

(3) If $f$ is $(P)$ integrable on $E$, and $E_{0}<E$, then $f$ is also $(P)$ integrable on $E_{0}$.

This holds, if we restrict ourselves to $(Y)$ integrals, only in case both $E_{0}$ and $E$ are measurable.

On account of the fact that, for a division of the set $E$ into sets measurable relative to $E$, we also have

$$
\text { meas } E=\Sigma_{i} \text { meas } E_{i} \text {, }
$$

we can obtain a definition of integration on sets $E$ by replacing, in the Pierpont integral, upper measure by lower measure. If we call integrability after the manner of this last definition $\left(P_{0}\right)$ integrability, then if $f(x)$ is $(P)$ integrable on $E$ it is also $\left(P_{0}\right)$ integrable. The converse does not seem to hold, because a set of lower measure zero does not necessarily have zero measure.

While the Pierpont integral gives an integral for a function defined on a non-measurable set, it is an extension of the

\footnotetext{
* Cf. Lamond, Transactions Amer. Math. Society, vol. 16 (1915), p. 393, where we find a similar theorem for $f$ bounded.
} 
Lebesgue definition only in a limited sense. For if we enclose any non-measurable set $E$ in a measurable set $M$ of such a nature that meas $M=\overline{\text { meas }} E$, then there exists on $M$ a function $f_{0}(x)$ measurable on $M$ and equal to $f(x)$ on $E$, such that

$$
\text { (L) } \int_{M} f_{0}(x)=(P) \int_{E} f(x) \text {; }
$$

i. e., a function $f(x)$ measurable relative to a set $E$ is a section of a measurable function defined on a measurable set including $E$. By using (4) of $\S 5$, we can construct the function $f_{0}(x)$ by taking the function $\varphi$ of $\S 2$, corresponding to $f$, but defined on $M$, and modifying it at the points of $E$ of measure zero, in such a way that it agrees with $f$ on $E$.

We gain insight into the Pierpont integral also from the geometrical point of view. If $f>0$, and we consider the planar set $[x$ on $E, 0 \leqq y<f(x)$ ], then the $(P)$ integral gives us the upper planar measure of this set, the $\left(P_{0}\right)$ integral the lower planar measure. These will be equal for $f>0$ only if the set $E$ for which $f>0$ is measurable, i. e., $f$ is Lebesgue integrable.

The $(P)$ integral has proved of value in connection with the reduction of a double integral to an iterated integral, in so far as a linear section of a planar measurable set is not necessarily linearly measurable.

It seems, however, that the question of giving a definition for the integration of essentially non-measurable functions, i. e., for instance functions defined on a measurable set which are not measurable, has not yet been satisfactorily solved.

7. The Pierpont Definition of Integration for Unbounded Functions.-(Cf. (8), pages $405 \mathrm{ff}$.) We note finally Pierpont's treatment of integrals of functions which are not bounded on a set $E$, very briefly. Let $E_{\alpha \beta}(\alpha, \beta>0)$ be the subset of $E$ for which $-\beta \leqq f(x) \leqq \alpha$. Suppose that the (P) $\int_{E_{\alpha \beta}} f(x) d x$ exists for every $\alpha, \beta>0$. Then $\int_{E}$ is defined to be the

$$
\lim _{\alpha, \beta=\infty} \int_{E_{\alpha \beta}}
$$

provided this limit exists and has a finite value. 
This definition is equivalent to the one given above in $\S 6$. For, if we assume that $(P) \int_{E} f$ exists, then $f$ will be relatively measurable on $E$, and hence $(P) \int_{E_{\alpha \beta}}$ will exist for every $\alpha$ and $\beta$. We show that

$$
\lim _{a, \beta=\infty}(P) \int_{E_{\alpha \beta}}=(P) \int_{E}
$$

by using the property (2) of $\S 6$. On the other hand if (P) $\int_{E_{\alpha \beta}}$ exists for every $\alpha, \beta$, then we can show that the $E_{\alpha \beta}$ are measurable relative to $E$, and to each other by using property (3) of $\S 5$, from which we conclude that $f$ is measurable relative to $E$. The existence of

$$
\text { (P) } \int_{E}=\lim _{\alpha, \beta} \int_{E_{\alpha \beta} \text { । }}
$$

follows from property (2) of $\$ 6$.

II. The Borel and Denjoy Definitions of Integration.

Suppose that, in an interval $(a, b), f(x)$ is not bounded in every vicinity of a point $c$; suppose further that the definite (Riemann) integral exists in the intervals $\left(a, c-\epsilon^{\prime}\right)$ and $\left(c+\epsilon^{\prime \prime}, b\right)$ for every $\epsilon^{\prime}$ and $\epsilon^{\prime \prime}$. Then consistent with the continuity of the definite integral as a function of its limits we define (as suggested by Cauchy)

$$
\int_{a}^{b} f(x)=\lim _{\boldsymbol{e}^{\prime}=0} \int_{a}^{c-\mathrm{e}^{\prime}} f(x)+\lim _{\mathbf{e}^{\prime \prime} \doteq 0} \int_{c+\mathrm{e}^{\prime \prime}}^{b} f(x) .
$$

Evidently the extension of this to the case in which the number of points of $(a, b)$ in every vicinity of which $f(x)$ is not bounded is finite, is immediate.

When the number of points in the vicinity of which $f(x)$ is not bounded becomes infinite then there are two types of definition. One of them gives a definition by means of a single limiting process, the other by a denumerable set of such processes. The first of these leads to the HarnackJordan-Moore and Borel types of integration, the other to the Dirichlet, extended by Hoelder and Lebesgue, and Denjoy definitions of integration. 
1. The Harnack-Jordan Definition.-(Cf. Harnack (9), pages $220 \mathrm{ff}$; Jordan (12), pages 50, 51; Moore (15); Nalli (16), pages 20-27.) We note first of all that the set of points in every vicinity of which $f(x)$ is not bounded constitute a closed set. We call this set for the present the set of singularities, and denote it by $Z$. Then Harnack defines the integral of $f(x)$ as follows:

Suppose the set $Z$ of singularities of $f(x)$ is of content zero. Enclose them in a finite set of intervals of total length $\epsilon$. Let $f_{1}(x)$ be zero in the interior of the enclosing intervals, and equal to $f(x)$ everywhere else and suppose that $\int_{a}^{b} f_{1}(x) d x$ exists. If this integral approaches a finite limit as $\epsilon$ approaches zero, this limit is said to be the integral of $f(x)$ from $a$ to $b$.

Jordan gives a definition which is equivalent to this in case the content of $Z$ is zero. His definition is as follows:

Divide $(a, b)$ into any finite number of intervals of maximum length $\delta$. Exclude the intervals containing points of the set $Z$, and suppose that the (Riemann) integrals of $f(x)$ exist on the remaining intervals. If the sum of these integrals approaches a definite finite limit when $\delta$ approaches zero, this is defined to be the integral of $f(x)$ from $a$ to $b$.

Moore ((15), pages 300-302) observes that the Harnack definition can be applied when the set $Z$ is replaced by another $Z_{0}$ containing it, and that the resulting integral is in reality a function of the set $Z_{0}$. Further, that in case the set of singularities $Z$ is non-existent then the integral of $f(x)$ on the basis of the set $Z_{0}$ is equal to the ordinary integral of $f(x)$ if and only if the set $Z_{0}$ is of content zero. For that reason it is desirable to restrict the consideration of these integrals to sets $Z$ of content zero, and it is to be supposed that Jordan assumed this to be the case even though he does not state this fact explicitly.*

2. The Borel Integral.-(Cf. Borel (2a), pages 199-205; (2b), pages 249-252; Hahn (8).) Borel has given a definition similar to that of Harnack except that he assumes that the set of singularities $Z$ is of measure zero, and may be everywhere dense, i. e., in general not closed. In this case $Z$ is not necessarily the set of all points in every neighborhood of which the function $f(x)$ becomes unbounded.

\footnotetext{
* No change, however, is made in the definition in the $3 \mathrm{~d}$ edition of his Cours d'Analyse.
} 
Before taking up the definition which Borel gives, it may be desirable to observe that it is possible to define a Riemann integral of a function $f(x)$ on a perfect or closed set $P$. Viz., if in the sum

$$
\sum_{i=1}^{n} f\left(\xi_{i}\right)\left(x_{i}-x_{i-1}\right)
$$

we replace the length of the interval $\left(x_{i}, x_{i-1}\right)$ by the content $h_{i}$ of the part of $P$ contained in the interval, and choose $\xi_{i}$ to belong to the $i$ th interval and $P$, then the limit of this sum as the length of the maximum subinterval approaches zero is the Riemann integral of $f(x)$ on $P$. We note that a necessary and sufficient condition for the existence of a Riemann integral on $P$ is that the set of points of $P$ at which $f(x)$ is discontinuous of measure be zero.

Borel's definition is then equivalent to the following

Definition. Suppose the set $Z$ of singularities is of measure zero, and such that if it is enclosed in a set of intervals of total length $\epsilon$, each interval containing at least one point of the set $Z$, then the Riemann integral of $f(x)$ exists on the complementary set $P_{\mathrm{e}}$. If

$$
\lim _{\epsilon=0} \int_{P_{\varepsilon}} f(x)
$$

exists, for all possible enclosures of the type described, it is defined to be the $(B)$ integral of $f(x)$ on $(a, b)$.

Borel assumes that this definition is to be applied to functions which are not bounded on $(a, b)$, but it can obviously be applied also to functions which are bounded. The relation of this definition to the one of Lebesgue has been discussed by Hahn (8). He proves the following theorems:

(1) If $f(x)$ is absolutely $(B)$ integrable, i. e., if $(B) \int|f(x)| d x$ exists, then $f(x)$ is also Lebesgue integrable.

Apparently this includes as a special case that in which $f(x)$ is a bounded function.

By way of proof we note that $(R) \int_{P_{e}}=(L) \int_{P_{e}}$ for any $f$ for which the first integral exists. Let now $\epsilon_{1}, \cdots, \epsilon_{n}, \cdots$, be such that $\lim _{n} \epsilon_{n}=0$, and let $P_{n}=P_{\epsilon_{n}}$ be the corresponding closed sets of measure greater than $b-a-\epsilon_{n}$, which are chosen in such a way that $P_{n}$ contains $P_{n-1}$. If then $f_{n}=f$ on $P_{n}$ and 
zero everywhere else, then the $\left|f_{n}\right|$ will form a monotonic nondecreasing sequence of functions approaching $|f|$ except possibly at a set of measure zero. Hence by the properties of $(L)$ integrals

(B) $\int_{a}^{b}|f|=\lim _{n} \int_{P_{n}}|f|=\lim _{n}(L) \int_{P_{n}}|f|$ $=\lim _{n}(L) \int_{a}^{b}\left|f_{n}\right|=(L) \int_{a}^{b}|f|$.

From this we conclude the existence of $(L) \int_{a}^{b} f$ and since meas $P_{\epsilon}$ approaches $b-a$, its equality to $(B) \int_{a}^{b} f$.

(2) A necessary and sufficient condition under the hypothesis of the definition that $f$ be $(B)$ integrable is that $(a) f$ be $(L)$ integrable, i. e., summable, on the set $Z+Z^{\prime}$, where $Z^{\prime}$ is the derived set of $Z$; and $(b)$, if $u_{n}=\left(a_{n}, b_{n}\right)$ are the intervals complementary to the closed set $Z+Z^{\prime}$, and $W\left(u_{n}\right)$ is the maximum value of $\left|\int_{u_{n^{\prime}}} f\right|$ for all intervals $u_{n}{ }^{\prime}$ interior to $u_{n}$, then $\Sigma_{n} W\left(u_{n}\right)$ shall form a convergent series. Moreover

where

$$
\text { (B) } \int_{a}^{a b} f(x) d x=\Sigma_{n} \int_{u_{n}} f(x) d x+(L) \int_{Z+Z^{\prime}} f(x) d x,
$$

$$
\int_{u_{n}}=\lim _{u_{n^{\prime}} \dot{=} u_{n}} \int_{u_{n^{\prime}}} \text {. }
$$

We refer to the original memoir of Hahn for the proof of this theorem. In case the set $Z+Z^{\prime}$ is of measure zero, and therefore of content zero, the Borel definition may be reduced to that of Harnack-Moore and the above equality lacks the second term on the right, in which form it was originally derived by Moore (cf. (15), pages $324 \mathrm{ff}$.). We shall refer to this theorem as the Moore-Hahn theorem.

(3) The Borel definition is not equivalent to the Lebesgue definition of integration, in particular there exist functions which are Borel integrable without being Lebesgue integrable, and functions which are Lebesgue integrable without being Borel integrable. 
For, as given, the definition of Borel includes functions which are not absolutely integrable (cf. Moore (15), page 327), while any Lebesgue integrable function is absolutely integrable. On the other hand the function $f(x)$, which is zero except at the points of a non-dense perfect set of measure greater than zero, at which it has the value 1 , though $(L)$ integrable, is not $(B)$ integrable. For Hahn has shown that there does not exist any set of measure zero such that for every enclosure of this set, each interval of the enclosure containing a point of this set, the function $f(x)$ is Riemann integrable on the set of points complementary to the intervals.

We can give a definition which will include as special cases the Lebesgue and Borel integrals by assuming that $f(x)$ is bounded and Lebesgue integrable on the sets $P_{\mathrm{e}}$ and that the integral on the interval is the limit of the integrals on the sets $P_{\mathrm{e}}$. Then a theorem similar to the Moore-Hahn theorem will hold, the integrals $\int_{u_{n^{\prime}}}$ being now taken in the sense of Lebesgue.

A further extension is to assume that on the sets $P_{e}, f(x)$ is not necessarily bounded, but only Lebesgue summable. That this actually is an extension can be shown by a simple example.

Suppose that the interval is $(0,1)$ and $f(x)$ is defined as follows: (a) in the interval $i_{n}=\left(\frac{1}{n}, \frac{1}{n+1}\right), f(x)>0$ if $n$ is even and $f(x)<0$ if $n$ is odd;

$$
\left|\int_{i_{n}} f(x) d x\right|=\frac{1}{n}
$$

(c) $f(x)$ is continuous throughout $i_{n}$ except that

$$
\lim _{\epsilon \doteq 0}\left|f\left(\frac{1}{n}+\epsilon\right)\right|=\infty \text {. }
$$

Then it can be shown* that the Borel integral of this function in the interval $(0,1)$ does not exist, the set of singularities being the points $\frac{1}{2}, \frac{1}{3}, \frac{1}{4}, \cdots, 0$; if, however, we apply the last definition suggested above with the set of singularities $Z=(0)$, we obtain an integral.

\footnotetext{
* Cf., for instance, Moore (15), p. 329.
} 
We note finally that the Harnack-Borel integrals do not have the property which we find in the case of Lebesgue and Riemann integrals that if $f$ and $g$ are integrable, then $f+g$ is also integrable. Borel has suggested that in such a case we set by definition

$$
\int(f+g)=\int f+\int g .
$$

3. The $\left(B^{*}\right)$ Integral.-(Cf. Hahn (8), page 9; Nalli (16), pages 84-97.) In the Borel definition of integral we note $(a)$ that the set of singularities $Z$ is a fixed set of points (i. e., in a way the integral is a function of the set $Z)$; (b) every allowable set of intervals is such that each interval of the set contains at least one point of the set $Z$. A definition has been suggested* in which both of these requirements are dropped. It is as follows:

Definition. Suppose (a) $f(x)$ is such that for every $\epsilon>0$, there exists a perfect set $P_{\mathrm{e}}$ of measure greater than $b-a-\epsilon_{\text {, }}$ for which the Riemann integral $\int_{P_{e}} f$ exists; further suppose (b) that

$$
\lim _{\boldsymbol{e} \doteq 0} \int_{P_{e}} f
$$

exists. Then this limit is said to be the $\left(B^{*}\right)$ integral of $f(x)$ on $(a, b)$.

Obviously since for every $\epsilon$ we can find in any measurable set $E$ a perfect set $P_{\epsilon}$ such that meas $E-$ meas $P_{\epsilon}<\epsilon$ we can apply the same type of definition to the definition of an integral extended over a measurable set $E$.

We note the following propositions:

(1) A necessary and sufficient condition that there exist a $\left(B^{*}\right)$ integral is that for every $\epsilon>0$ there exists a $\delta_{\epsilon}$ such that if $P_{1}$ and $P_{2}$ have measures greater than $b-a-\delta_{e}$, and $\int_{P_{1}}$ and $\int_{P_{2}}$ exist, then

$$
\left|\int_{P_{1}}-\int_{P_{2}}\right| \leqq \epsilon .
$$

(2) If the $\left(B^{*}\right)$ integral of $f(x)$ exists on $(a, b)$, then for any sequence of $\epsilon$ 's such that $\epsilon_{1} \geqq \epsilon_{2} \geqq \cdots$ and $\lim _{n} \epsilon_{n}=0$

* Cf. Hahn (8), p. 9. Nalli (16), p. 85, erroneously asserts that Borel's definition is the one which we now give. 
we can find a sequence of perfect sets $P_{n}$ of measure greater than $b-a-\epsilon_{n}$, each containing the preceding, such that $\int_{P_{n}}$ exists and $\lim _{n} \int_{P_{n}}=\left(B^{*}\right) \int_{a}^{b}$.

For this purpose we obtain a series of $P_{\epsilon}: P_{e_{1}-e_{2}}, P_{\epsilon_{2}-e_{3}}, \cdots$. For $P_{n}$ we take the greatest perfect set common to the sets $P_{\epsilon_{m}-\epsilon_{m+1}}$, for $m \geqq n$. This will evidently have measure greater than $b-a-\epsilon_{n}$, and be contained in $P_{n+1}$.

(3) If $f(x)$ is bounded in $(a, b)$ and fulfils condition $(a)$ of the definition, then the $\left(B^{*}\right)$ integral exists.

(4) If $f$ and $g$ are $\left(B^{*}\right)$ integrable, then $f+g$ is $\left(B^{*}\right)$ integrable and we have

$$
\left(B^{*}\right) \int(f+g)=\left(B^{*}\right) \int f+\left(B^{*}\right) \int g,
$$

a proposition which does not hold for the $(B)$ integral.

(5) If $f$ is integrable according to the Lebesgue definition, then $f$ is also $\left(B^{*}\right)$ integrable, the values of the integrals being the same.

For if $f$ is $(L)$ integrable, then $f$ is measurable. From the theorem of Lusin* on measurable functions:

If $f$ is measurable in an interval $(a, b)$, then for every $\epsilon>0$ there exists a non-dense perfect set $P_{\mathbf{e}}$ of measure greater than $b-a-\epsilon$ on which $f$ is continuous,

it follows that the condition $(a)$ of the definition is satisfied, i. e., for every $\epsilon>0$ there exists a $P_{\mathrm{e}}$ of measure greater than $b-a-\epsilon$ for which the $(R) \int_{P_{\epsilon}}$ exists. Evidently

$$
\text { (R) } \int_{P_{e}}=(L) \int_{P_{e}} \text {. }
$$

Moreover we know that the $(L)$ integral is absolutely continuous, i. e., if $\lim _{n}$ meas $E_{n}=$ meas $E$, then $\lim _{n}(L) \int_{E_{n}}=(L) \int_{E}$. Hence since

it follows that

$$
\lim _{\epsilon=0} \operatorname{meas} P_{\epsilon}=b-a,
$$

* Cf. Comptes Rendus, vol. 154 (1912), p. 1689. Cf. also Lebesgue, Comptes Rendus, vol. 137 (1903), p. 1229. 


$$
\lim _{\epsilon \dot{=} 0}(R) \int_{P_{\epsilon}}=\lim _{\epsilon \dot{\epsilon}=0}(L) \int_{P_{\epsilon}}=(L) \int_{a}^{b},
$$

i. e., the $\left(B^{*}\right)$ integral exists and is equal to the $(L)$ integral. The same method of reasoning would show that if $(L) \int_{E}$ exists, where $E$ is any measurable set, then $\left(B^{*}\right) \int_{E}$ exists also.

We note in passing that the Hahn example of a function not $(B)$ integrable is obviously $\left(B^{*}\right)$ integrable.

On the other hand we also have

(6) If $f(x)$ is $\left(B^{*}\right)$ integrable on $(a, b)$, then it is also $(L)$ integrable.

We observe that if we show that an $f$ which is $\left(B^{*}\right)$ integrable is absolutely $\left(B^{*}\right)$ integrable, i. e., $\left(B^{*}\right) \int|f|$ exists, we can apply the method of proof used for (1) of $\S 2$ above, for the case of $(B)$ integrability. We therefore proceed to show

(7) If $f(x)$ is $\left(B^{*}\right)$ integrable then $|f|$ is also $\left(B^{*}\right)$ integrable. Suppose if possible that $|f|$ is not $\left(B^{*}\right)$ integrable. Then from (2) above it would follow that $\left(B^{*}\right) \int|f|$ is infinite. Hence if $f_{1}=\frac{1}{2}(|f|+f)$ and $f_{2}=\frac{1}{2}(|f|-f)$, then by (4) $\left(B^{*}\right) \int f_{1}$ and $\left(B^{*}\right) \int f_{2}$ are both infinite. For every $M$ we can then determine a perfect set $P_{1 \epsilon}$ of measure greater than $b-a-\epsilon$ such that $\int_{P_{1 \epsilon}} f_{1}>M$. For the same $\epsilon$ we can determine a perfect set $P_{2 \mathrm{e}}$ on which $(R) \int_{P_{2 \mathrm{e}}} f_{2}$ exists, and consequently $f_{2}$ is bounded on $P_{2 \epsilon}$. Suppose $f_{2}<m$ on $P_{2 \varepsilon}$. Then $(R) \int_{P_{2 \epsilon}}^{0} f_{2}$ $<m(b-a)$. Let the set $E$ be the sum of the measurable sets: (a) the subset of $P_{1 \mathrm{e}}$ for which $f_{1}>0$ and hence $f_{2}=0$; (b) the subset of $P_{2 \epsilon}$ for which $f_{2}>0 ;(c)$ the greatest common subset of $P_{1 \mathrm{e}}$ and $P_{2 \varepsilon}$. Then the measure of $E$ will be greater than $b-a-2 \epsilon$ and

$$
\text { (L) } \int_{E} f=(R) \int_{P_{1 \mathrm{e}}} f_{1}-(R) \int_{P_{\mathbf{2}}} f_{2}>M-m(b-a) .
$$

However, by (5) we can find a perfect set $P_{\mathrm{e}}$ of measure greater 
than or equal to $b-a-2 \epsilon$, contained in $E$, such that the difference between $(L) \int_{E} f$ and $(R) \int_{P_{\mathrm{e}}} f$ is as small as we please, i. e., such that

$$
\text { (R) } \int_{P_{\mathbf{e}}} f \geqq M-\dot{m}(b-a) .
$$

Since $M$ has been chosen arbitrarily, it follows that

$$
\lim _{\epsilon \doteq 0} \int_{P_{e}} f
$$

does not exist, i. e., $f$ is not $\left(B^{*}\right)$ integrable, which is a contradiction. Hence the theorem.

We thus have the result

(8) $\left(B^{*}\right)$ integrability is equivalent to $(L)$ integrability, the values of the integrals being the same.

The absolute convergence of the $\left(B^{*}\right)$ integral is another instance of a phenomenon observed by Stolz* and Moore. $\dagger$ In the $\left(B^{*}\right)$ integration while the singularities are enclosed in a set of intervals of total length less than $\epsilon$, it is not required that every interval contain a point of the set, i. e., we have a broad enclosure. On the other hand, in the $(B)$ integration, every interval must include a point of the set of singularities, i. e., we have a narrow enclosure. The broad enclosures yield absolutely convergent integrals, the narrow enclosures may lead to non-absolutely convergent integrals.

4. The Dirichlet-Lebesgue Extension of the Cauchy Definition.-(Cf. Lebesgue (13), pages 9-14.) The Cauchy definition of integral of a function $f(x)$, which has only a finite number of points in every neighborhood of which $f(x)$ is not bounded, can be extended so as to give the Harnack and Borel definitions of integration as a result of a single limiting process. We turn now to the other type of definition which requires a finite or denumerable infinitude of limiting processes.

The first step in this direction was taken by Dirichlet, who extends the Cauchy definition to the case in which the interval $(a, b)$ has a set of singularities $Z$ whose derived set $Z^{\prime}$ contains a finite number of points $x_{1}, \cdots, x_{n}$. For in any subinterval interior to the interval $\left(x_{i-1}, x_{i}\right)$ we have only a finite number of

* Wiener Berichte, vol. 108 $2 a$ (1899), p. 1235.

$\dagger(5)$, pp. 302-304, 323. 
singularities. To find $\int_{x_{i-1}-\mathrm{e}}^{x_{i}+\mathrm{e}^{\prime}} f(x) d x$, for any two positive $\epsilon$ and $\epsilon^{\prime}$, we can then apply the Cauchy definition. If the limit of this integral exists as $\epsilon$ and $\epsilon^{\prime}$ approach zero, it is the integral in the interval $\left(x_{i-1}, x_{i}\right)$, and the integral in $(a, b)$ is the sum of these integrals.

We observe that the Cauchy-Dirichlet definitional process makes use of the following two conditions:

(a) If an integral exists on each of a finite set of consecutive intervals $\left(a_{1}, a_{2}\right) ;\left(a_{2}, a_{3}\right) ; \cdots ;\left(a_{n-1}, a_{n}\right)$, then $\int_{a_{1}}^{a_{n}}=\sum_{i=1}^{n-1} \int_{a_{i}}^{a_{i+1}} \cdot$
(b) If $\int_{a+\mathrm{e}^{\prime}}^{b-\mathrm{e}^{\prime \prime}}$ exists for every $\epsilon^{\prime}$ and $\epsilon^{\prime \prime}$, and if this integral
approaches a limit as $\epsilon^{\prime}$ and $\epsilon^{\prime \prime}$ approach zero, this limit is deapproaches a limit as $\epsilon^{\prime}$ and $\epsilon^{\prime \prime}$ approach zero, this limit is defined to be $\int_{a}^{b}$.

Evidently the Dirichlet manner of reasoning is immediately extensible to the case in which $Z^{\prime \prime}$ and then $Z^{(a)}, \alpha$ being any finite or transfinite number of the first and second classes, contains a finite number of points, ${ }^{*}$ i. e., if $Z$ is a closed reducible set, then this method will give a definition of integration over the interval $(a, b)$. Lebesguef shows that this same result may be attained by the following definition:

A function $f(x)$ has an integral in the finite interval $(a, b)$ if there exists a continuous function $F(x)$, unique except for the addition of a constant, such that we have in every interval $(\alpha, \beta)$ where $f(x)$ is continuous

$$
\int_{\alpha}^{\beta} f(x) d x=F(\beta)-F(\alpha)
$$

$F(x)$ is the indefinite integral of $f(x)$ and we have

$$
\int_{a}^{b} f(x) d x=F(b)-F(a) .
$$

In applying the process just discussed we start from $Z^{(a)}$, and work back to $Z$ by a denumerable set of limiting proc-

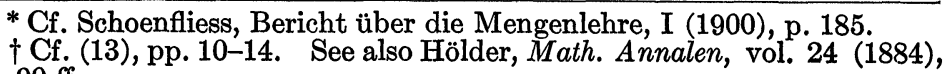
pp. 90 ff. 
esses, in every interval into which $Z^{(a)}$ divides $(a, b)$. We can obtain the same result in case $Z$ is reducible, by starting with $Z$ and working up to $Z^{(a)}$. For consider the intervals complementary to $Z^{\prime}$. By the two conditions of the CauchyDirichlet process, we can then define an integral in each of these intervals. Evidently the same holds true of the intervals complementary to $Z^{\prime \prime}$, and so on. By a well-known theorem in the theory of point sets* we arrive finally at the intervals complementary to a set $Z^{(a)}$ which is either zero or perfect. If it is zero, then we have an integral for the entire interval. If perfect, then we have obtained an integral in each interval complementary to this perfect set $Z^{(\alpha)}=P$. If $W\left(u_{n}\right)$ is the maximum absolute oscillation of $\int_{u_{n}{ }^{\prime}}, u_{n}{ }^{\prime}$ being any interval interior to $u_{n}$, and $\Sigma W\left(u_{n}\right)$ is convergent, and $P$ is of content zero, then the Moore-Hahn theorem (2) of $\S 2$ would suggest that we define

$$
\int_{a}^{b} f(x) d x=\sum_{n=1}^{\infty} \int_{u_{n}} f(x) d x .
$$

We observe that this will include the Harnack integral as a special case. $\dagger$

We might proceed to a discussion of the case in which the content of $P$ is not zero, or the series of oscillations or integrals over the complementary intervals $u_{n}$ is not absolutely convergent, but we prefer to take up briefly the Denjoy definition of integration which combines the two definitional conditions of Cauchy-Dirichlet with the Moore-Hahn theorem, and includes as special cases almost all of the definitions so far considered in this chapter.

5. The Denjoy Definition of Integration.-(Cf. Denjoy (3); Nalli (16), pages $122 \mathrm{ff}$.) We give first a few definitions.

(a) A function $f(x)$ is said to be not summable at a point $x_{0}$ of an interval, if $f(x)$ is not summable in every interval containing the point $x_{0}$. Similarly $f(x)$ is not summable at a point $x_{0}$ on a perfect set $P$, if the function $\varphi=f$ on $P$ and zero at points not on $P$ is not summable at $x_{0}$. In either case the points at which $f(x)$ is not summable form a closed set.

(b) Let $\left[u_{n}\right]$ be the set of intervals complementary to a perfect set $P$, relative to an interval $(a, b)$, and suppose that

${ }^{*}$ Cf. Hobson, Theory of Functions, pp. 92, 93.

$\dagger$ Cf. Moore (15), p. 330. 
for every $n$ there is defined a constant $W\left(u_{n}\right)$. Then the series $\Sigma W\left(u_{n}\right)$ is said to be absolutely convergent on an interval $\delta$ if the part of the series $\Sigma W\left(u_{n}\right)$ which corresponds to the intervals interior to $\delta$ is absolutely convergent. It is absolutely convergent at a point $x_{0}$ of the perfect set $P$ if there exists an interval containing $x_{0}$ in which $\Sigma_{n} W\left(u_{n}\right)$ is absolutely convergent. Evidently the points of $P$ at which $\Sigma_{n} W\left(u_{n}\right)$ is not absolutely convergent form a closed set.

Then Denjoy defines an integral $V(a, b)$ on the interval $(a, b)$ as follows:

$(A)$ The integral of $f(x)$ in an interval in which $f(x)$ is summable is the Lebesgue integral.

$(B)$ If the integral has been defined for a finite number of consecutive intervals: $\left(a_{1}, a_{2}\right) ;\left(a_{2}, a_{3}\right) ; \cdots ;\left(a_{n-1}, a_{n}\right)$ then $V\left(a_{1}, a_{n}\right)$ is defined to be

$$
V\left(a_{1}, a_{n}\right)=\sum_{i=1}^{n-1} V\left(a_{i}, a_{i+1}\right) .
$$

(C) If (a) $f(x)$ is summable on a perfect set $P$, whose complementary intervals relative to an interval $(c, d)$ are $u_{n}$; (b) $V\left(u_{n}^{\prime}\right)$ has been defined on every interval $u_{n}^{\prime}$ containing no points of $P$ in its interior and $W\left(u_{n}\right)$ is the least upper bound of $\left|V\left(u_{n}^{\prime}\right)\right|$ for all possible $u_{n}^{\prime}$ interior to $u_{n}$; and (c) $\Sigma_{n} W\left(u_{n}\right)$ is convergent, then

$$
V(c, d)=\Sigma_{n} V\left(u_{n}\right)+\int_{P} f(x) d x .
$$

The function $f(x)$ is said to be Denjoy (or $(D n)$ ) integrable, if the following conditions are fulfilled:

I. The set of points of any perfect set $P$ at which $f(x)$ is not summable on $P$ is not dense on $P$.

II. If $V\left(c^{\prime}, d^{\prime}\right)$ has been defined for every interval $\left(c^{\prime}, d^{\prime}\right)$ interior to $V(c, d)$, then

$$
\lim _{c \doteq c^{\prime}, d \doteq d^{\prime}} V\left(c^{\prime}, d^{\prime}\right)
$$

exists and is defined to be $V(c, d)$.

III. The set of points of any perfect set $P$ at which the series of maximum oscillations $W\left(u_{n}\right)$ of $V$ on the intervals complementary to $P$ is not absolutely convergent is not dense on $P$.

The proof that these three definitional conditions and the 
three integrability conditions suffice to determine for a function $f(x)$ a finite number, its integral, is rather long. We mention only the chief features of the proof.

A central position is occupied by the theorem on point sets:

If we have a series of closed sets $E^{(a)}$, corresponding to the transfinite numbers of the first and second class, each contained in the preceding and not dense on it, then there exists a transfinite number $\alpha$ with a precedent, such that $E^{(a-1)} \neq 0$ and $E^{(a)}=0$.

Condition I when applied to the interval $(a, b)$ gives a closed set $E_{1}$, not dense on $(a, b)$ at every point of which $f$ is not summable on $(a, b)$. We break up $E_{1}$ into $P_{1}+R_{1}$, where $P_{1}$ is perfect and $R_{1}$ is reducible. Then we replace $(a, b)$ by $P_{1}$ and apply condition I again. This yields $E_{2}=P_{2}+R_{2}$. And so we continue. The theorem just given shows that this process must terminate after a denumerably infinite number of steps.

Condition II, with the definitional condition $B$, when applied to the intervals complementary to $E_{1}$ gives a value for the intervals complementary to the derived set $E_{1}{ }^{\prime}$ of $E_{1}$. The same two conditions lead us to the intervals complementary to $E_{1}{ }^{\prime \prime}$ and eventually to the intervals complementary to $P_{1}$.

In the same way, definitional condition $C$ together with the conditions II and III give us a value for $V(g, h)$ in any interval $(g, h)$ which contains points of $P_{1}$ but not of $E_{2}$, i. e., in any interval complementary to $E_{2}$. Similarly we find the value of $V(g, h)$ for every interval complementary to $E_{a+1}$, the values in the intervals complementary to $P_{a}$ having been determined.

We thus obtain a value for the integral $V(a, b)$ in the interval $(a, b)$ after a denumerably infinite number of steps.

We note the following properties:

(1) If $f(x)$ is such that $|f(x)|$ is Denjoy integrable, then $f(x)$ is Lebesgue integrable.

For evidently if $f(x) \geqq 0$, and there existed a point at which $f(x)$ were not summable on $(a, b)$, then $V(a, b)$ would also be infinite.

(2) From the Moore-Hahn theorem of $\S 2$ it follows that if $f(x)$ is $(B)$ integrable it is also Denjoy integrable, the definitional condition $C$ being an immediate extension of the expression for the $(B)$ integral. The same result holds true 
if in the $(B)$ integral we replace the Riemann integrals by Lebesgue integrals. Just how the definitions compare when Riemann integrability on $P_{e}$ is replaced by Lebesgue summability, we do not know, except that there are functions which are Denjoy integrable without being $(B)$ integrable in any extensions of the definition, an immediate consequence of

(3) If $f$ and $g$ are Denjoy integrable, then $f+g$ is also Denjoy integrable, and we have

$$
(D n) \int f+(D n) \int g=(D n) \int(f+g) .
$$

We have further

(4) The indefinite Denjoy integral $V(a, x)$ is a continuous function and has as derivative $f(x)$ except for a set of measure zero.

(5) If the continuous function $F(x)$ has at every point a finite derivative $f(x)$ then this derivative is Denjoy integrable, and the Denjoy integral of $f(x)$ has the value $F(x)-F(a)$.

This result is of importance in the theory of the derivatives of continuous functions. In the case in which the derivative is finite at every point, it gives a means of returning from the derivative to the original function. For the Riemann integral, the derived function $f(x)$ must be Riemann integrable; for the Lebesgue integral, $f(x)$ must be summable, and this carries with it that $F(x)$ be of bounded variation; but $f(x)$, finite at every point, is Denjoy integrable.*

For the proofs of these theorems we refer to the Denjoy articles, as well as the Nalli thesis, which contains a careful analysis of the Denjoy first notes on the subject.

Quite recently Khintchinet has pointed out that in order to obtain an integral by the Denjoy method of definition, it is sufficient to replace the condition of convergence of the maximum oscillations $W\left(u_{n}\right)$ in the intervals $u_{n}$, in the definitional condition $C$ and the integrability condition III, by the weaker condition of convergence of the series of variations of the integrals $\Sigma_{n} V\left(u_{n}\right)$, in as much as $\Sigma_{n} V\left(u_{n}\right)$ and not $\Sigma_{n} W\left(u_{n}\right)$

* Cf. in this connection Lusin: Comptes Rendus, volume 155 (1912), pp. 1475-7. See also Annali di Matematica, ser. 3, vol. 26 (1917), pp. 77131. Pp. 118-131 contain a very interesting suggestion for extending the definition of integration by the use of Fourier series.

† "Sur une extension de l'intégrale de M. Denjoy," Comptes Rendus, vol. 162 (1916), pp. 287-291. Cf. also pp. 377-380. 
appears in the integration expression. However, for an integral defined in this way it is no longer true that the derivative of the indefinite integral $V(a, x)$ is the function $f(x)$ except for a set of measure zero. By defining the term "integrals approaching zero asymptotically at a point $x_{0}$ of a perfect set $P$ " to mean that if $d_{n}$ is the distance between $x_{0}$ and the nearest extremity of the interval $u_{n}$ complementary to $P$, then

$$
\lim _{n} \frac{W\left(u_{n}\right)}{d_{n}}=0,
$$

he shows that a necessary and sufficient condition that the derivative of $V(a, x)$ be $f(x)$ except for a set of measure zero, is that the definitional condition $C$ be replaced by

$C^{\prime}$. If $f$ is summable on $P$ in an interval $(c, d)$, if, moreover, the integrals approach zero asymptotically except for a set of measure zero on $P$, then

$$
V(c, d)=(L) \int_{P} f+\Sigma_{n} V\left(u_{n}\right) ;
$$

and the integrability condition III by

III'. For every perfect set $P$, there exists a portion* $\pi$ of $P$ such that if $\delta_{1}, \cdots, \delta_{n}, \cdots$ are the intervals complementary to $\pi$, the series $\Sigma_{n} V\left(\delta_{n}\right)$ converges and the integrals approach zero asymptotically on $\pi$ except for a set of measure zero on $\pi$.

Both of the Khintchine suggestions give actual extensions of the Denjoy definition, i. e., give an integral for a function not integrable by the definition as given by Denjoy.

* Cf., for instance, Denjoy (4(a)), p. 120.

(To be continued.) 